\title{
Philosophy of CRISPR-Cas: Introduction to Eugene Koonin's target paper and commentaries
}

\author{
Thomas Pradeu ${ }^{1,2}$
}

Published online: 13 February 2019

(c) Springer Nature B.V. 2019

We are most grateful to Eugene Koonin for having accepted to write for Biology \& Philosophy a target paper on such a major topic in current biology as CRISPRCas (CRISPR-Cas stands for "Clustered Regularly Interspaced Short Palindromic Repeats"). There is indeed little doubt that the characterization of the CRISPR-Cas systems and their mechanisms constitutes a ground-breaking discovery in recent biological and biomedical sciences, from basic microbiology to technological applications (Doudna and Charpentier 2014). One sign of recognition among many has come from the leading journal Science, which chose CRISPR-Cas as its 2015 "breakthrough of the year" (McNutt 2015), described as "poised to revolutionize research" because of its role in genome editing.

In the beginning of the 2000s, CRISPR-Cas was identified as a form of immunity in Archaea and bacteria (Mojica et al. 2005; Pourcel et al. 2005; Bolotin et al. 2005; Makarova et al. 2006; Barrangou et al. 2007). This fundamental work followed decades of investigations, by many researchers from several laboratories around the world, that partly anticipated this discovery, making the history of CRISPR-Cas particularly intricate (Morange 2015a, b). In a nutshell, CRISPR-Cas makes it possible for many prokaryotes to detect and eliminate viruses (known as phages, in the case of bacteria) and to respond more efficiently if the same virus is encountered for a second time. Taken together, these results led to the now commonly accepted concept that CRISPR-Cas is a prokaryotic adaptive immune system, endowed with a form of immune "memory" (Hille et al. 2018).

More recently, CRISPR-Cas has been developed into an arsenal of novel, powerful tools for genome manipulation (Cong et al. 2013; Doudna and Charpentier 2014; Komor et al. 2017). By now, CRISPR-Cas has been widely adopted as a gene-editing tool in labs across the world, and many students learn how to use it. Yet, this fantastic toolkit also raises several important issues. First, many researchers are concerned

Thomas Pradeu

thomas.pradeu@u-bordeaux.fr

1 ImmunoConcept, UMR5164, CNRS \& University of Bordeaux, Bordeaux, France

2 Institut d'histoire et de philosophie des sciences et des techniques (IHPST), UMR8590, Paris, France 
about the potentially dramatic consequences of misguided use of CRISPR-Cas, especially in the situation when it could alter human DNA in a heritable way. At an international meeting in Napa, California, in 2015, a group of experts, including the world-leading CRISPR researcher Jennifer Doudna, called for a moratorium on using CRISPR-Cas9 technology for any modification of the germline genome in humans (Baltimore et al. 2015). In 2016, an advisory committee at the US National Institutes of Health $(\mathrm{NIH})$ approved the first human trial to use CRISPR gene editing. The goal of this trial is to edit the patient's own T cells via CRISPR-Cas9 technology as a part of a cancer therapy protocol (Reardon 2016). Second, part of the CRISPR-Cas research community has been plagued with disagreements over patents, with huge financial interests (Contreras and Sherkow 2017; Ledford 2018). It comes as no surprise, therefore, that, at present, CRISPR-Cas technologies are actively discussed by bioethicists, regulatory bodies, and the public more generally [e.g., (Belluck 2017)].

Eugene Koonin is the ideal person for presenting CRISPR-Cas to a philosophical audience. Koonin is a prominent biologist and an expert in evolutionary and computational biology. His research spans an expansive range of subjects, from genome sequencing to comparative genomics, protein classification, horizontal gene transfer (and related issues around the "tree of life"), identification of giant viruses, and virus classification, among many other areas. Koonin's work also has a strong conceptual and philosophical dimension, as illustrated by his book, The Logic of Chance (Koonin 2011), many of his scientific papers [e.g., (Koonin and Wolf 2010; Koonin and Krupovic 2015)], and also by his publications in philosophy of science journals [e.g., (Koonin and Starokadomskyy 2016)] or in collaboration with philosophers (O’Malley and Koonin 2011). Furthermore, and most crucially for the present issue of Biology \& Philosophy, Koonin has been a major actor in the discovery, characterization, and classification of CRISPR-Cas systems [e.g., (Makarova et al. 2001a, b2002, 2006, 2015; Shmakov et al. 2015; Mohanraju et al. 2016; Koonin et al. 2017)].

In the target paper presented in this issue and entitled "CRISPR: a new principle of genome engineering linked to conceptual shifts in evolutionary biology", Koonin argues that CRISPR-Cas systems offer to date the best instance of a mechanism of Lamarckian evolution. He defines such Lamarckian evolution as the generation of heritable, adaptive genomic changes in response to encounters with external factors (he also talks about "Inheritance of Acquired adaptive Characteristics", or "IAC"). In the case of CRISPR-Cas, the external factor corresponds to foreign nucleic acids (phages, plasmids, and so on). He describes the CRISPR-Cas system in prokaryotes as an adaptive immune system, based on self-nonself discrimination. He also examines the connections between CRISPR-Cas and programmed cell death and dormancy induction in microbes, two phenomena possibly associated with a form of evolutionary "altruism". Finally, he explores the role that mobile genetic elements (MGE) played in the origin of various components of CRISPR-Cas systems.

Koonin's paper is followed by a series of selected commentaries, written by biologists of different backgrounds and philosophers of biology. In the first commentary, "Mutationism, not Lamarckism, captures the novelty of CRISPR-Cas", Jeremy Wideman, Andrew Inkpen, Ford Doolittle, and Rosemary Redfield (Wideman et al. 
2018) reject Koonin's characterization of CRISPR-Cas as a Lamarckian mode of evolution. Instead, they suggest that the CRISPR-Cas system is better conceptualized as a process of directed mutations, that is, mutations (rather complex ones in the case of CRISPR-Cas, because they are based on spacer acquisition) that are not random with respect to the potential benefit. In their view, such a description enables one to situate CRISPR-Cas more accurately within debates between mutationists and neo-Darwinists, ${ }^{1}$ as it constitutes a strong objection against the neo-Darwinist view that the cause of a new mutation is not influenced by its benefit or cost, rather than in the context of Lamarck's view in the early 1800s.

In another quite critical commentary, entitled "Striving for clarity about the "Lamarckian" nature of CRISPR-Cas systems", Sam Woolley, Emily C. Parke, David Kelley, Anthony M. Poole, and Austen Ganley (Woolley et al. 2018) make three main claims against the views expressed in Koonin's paper. First, according to the authors, Koonin sometimes makes a confusion between the system of CRISPRCas as such and the mechanism that it uses. For them, an accurate description is that the CRISPR-Cas system uses two mechanisms (acquisition of pathogen DNA and transcription/targeting), and the only question is whether the mechanism of acquisition of pathogen DNA is Lamarckian or not. Second, after distinguishing three meanings of "Lamarckism", they reject the view that the CRISPR-Cas system would be "Lamarckian", particularly because it does not exhibit the kind of teleological connotation usually associated by Lamarckism. Finally, they propose that a key feature of CRISPR-Cas systems is that they do hold the potential for a genuinely nonDarwinian, directed evolution, but one that differs from what Koonin describes, and that is based instead on the use of CRISPR-Cas as a human gene-editing tool.

In "A Use/Disuse Paradigm for CRISPR-Cas Systems", Sophie Veigl (2018) narrows down the criteria for counting as a "Lamarckian" phenomenon, and then proposes that interference-driven spacer acquisition (IDSA) could be seen as an instantiation of a truly Lamarckian paradigm. Furthermore, she considers whether Lamarckian inheritance and canonical inheritance can be understood within the philosophical context of theoretical pluralism.

In her commentary, entitled "Lamarckian realities: the CRISPR-Cas system and beyond", Jablonka (2018) proposes to contextualize and extend Koonin's claim that the CRISPR-Cas system should be considered as "Lamarckian". She offers a conceptually and historically enriched characterization of "Lamarckism" and "quasiLamarckism" [see also (Jablonka and Gissis 2011)]. She agrees that CRISPR-Cas offers to date a prime example of a phenomenon of inheritance of acquired genomic characters. Additionally, she emphasizes how Lamarckian and quasi-Lamarckian processes, including CRISPR-Cas, influence evolvability.

In "CRISPR-Cas Changing Biology?", Baxter (2018) focuses on the human dimension of recent CRISPR-Cas research. She argues that CRISPR-Cas technologies are not "natural" genome editing systems, but, at least in part, human artefacts. Moreover, she emphasizes that, in a case such as CRISPR-Cas systems, the traditional distinction between fundamental and applied research is blurred.

\footnotetext{
${ }^{1}$ On these debates, see (Gayon 1998; Beatty 2016).
} 
Finally, Thomas Pradeu and Jean-François Moreau, in "CRISPR-Cas immunity: Beyond nonself and defence" (Pradeu and Moreau 2018), propose to adopt an extended view of CRISPR-Cas immunity by arguing that CRISPR-Cas includes, but cannot be reduced to, defence against nonself. First, CRISPR-Cas systems can target endogenous elements and tolerate exogenous elements. Second, CRISPR-Cas-based immunity concerns activities that go beyond defence, such as DNA repair. In being not reducible to immunity against nonself, CRISPR-Cas is similar to other forms of immunity across the living world.

This series of papers ends with Eugene Koonin's response to commentaries. In addressing in detail virtually all the remarks and objections made by the different commentators, Koonin offers an even richer and more precise picture of his views about CRISPR-Cas. We would like to thank once again Eugene Koonin for this inaugural target paper as well as all the commentators for their excellent work on a highly exciting topic in current biology which will undoubtedly continue to instigate vigorous debates among biologists and philosophers alike in the years to come.

Acknowledgments T. P. has received funding from the European Research Council (ERC) under the European Union's Horizon 2020 research and innovation programme - Grant Agreement no. 637647 - IDEM.

\section{References}

Baltimore D, Berg P, Botchan M et al (2015) A prudent path forward for genomic engineering and germline gene modification. Science 348:36-38. https://doi.org/10.1126/science.aab1028

Barrangou R, Fremaux C, Deveau H et al (2007) CRISPR provides acquired resistance against viruses in prokaryotes. Science 315:1709-1712. https://doi.org/10.1126/science.1138140

Baxter (2018) CRISPR-Cas changing biology? A commentary on 'CRISPR: a new principle of genome engineering linked to conceptual shifts in evolutionary biology'. Biol Philos. https://doi. org/10.1007/s10539-018-9658-7

Beatty J (2016) The creativity of natural selection? Part I: Darwin, Darwinism, and the mutationists. J Hist Biol 49:659-684. https://doi.org/10.1007/s10739-016-9456-5

Belluck P (2017) Scientists Repair a risky mutation. N. Y. Times, New York City (Aug 3, 2017)

Bolotin A, Quinquis B, Sorokin A, Ehrlich SD (2005) Clustered regularly interspaced short palindrome repeats (CRISPRs) have spacers of extrachromosomal origin. Microbiol Read Engl 151:2551-2561. https://doi.org/10.1099/mic.0.28048-0

Cong L, Ran FA, Cox D et al (2013) Multiplex genome engineering using CRISPR/Cas systems. Science. https://doi.org/10.1126/science.1231143

Contreras JL, Sherkow JS (2017) CRISPR, surrogate licensing, and scientific discovery. Science 355:698-700. https://doi.org/10.1126/science.aal4222

Doudna JA, Charpentier E (2014) The new frontier of genome engineering with CRISPR-Cas9. Science 346:1258096. https://doi.org/10.1126/science.1258096

Gayon J (1998) Darwinism's struggle for survival: heredity and the hypothesis of natural selection. Cambridge University Press, Cambridge

Hille F, Richter H, Wong SP et al (2018) The biology of CRISPR-Cas: backward and forward. Cell 172:1239-1259. https://doi.org/10.1016/j.cell.2017.11.032

Jablonka E (2018) Lamarckian realities: the CRISPR-Cas system and beyond. Biol Philos. https://doi. org/10.1007/s10539-018-9660-0

Jablonka E, Gissis S (2011) Transformations of lamarckism: from subtle fluids to molecular Biology. MIT Press, Cambridge 
Komor AC, Badran AH, Liu DR (2017) CRISPR-based technologies for the manipulation of eukaryotic genomes. Cell 168:20-36. https://doi.org/10.1016/j.cell.2016.10.044

Koonin EV (2011) The logic of chance: the nature and origin of biological evolution, 1st edn. FT Press, Upper Saddle River

Koonin EV, Krupovic M (2015) Evolution of adaptive immunity from transposable elements combined with innate immune systems. Nat Rev Genet 16:184-192. https://doi.org/10.1038/nrg3859

Koonin EV, Starokadomskyy P (2016) Are viruses alive? The replicator paradigm sheds decisive light on an old but misguided question. Stud Hist Philos Sci Part C Stud Hist Philos Biol Biomed Sci 59:125-134. https://doi.org/10.1016/j.shpsc.2016.02.016

Koonin EV, Wolf YI (2010) Constraints and plasticity in genome and molecular-phenome evolution. Nat Rev Genet 11:487-498. https://doi.org/10.1038/nrg2810

Koonin EV, Makarova KS, Zhang F (2017) Diversity, classification and evolution of CRISPR-Cas systems. Curr Opin Microbiol 37:67-78. https://doi.org/10.1016/j.mib.2017.05.008

Ledford H (2018) Pivotal CRISPR patent battle won by Broad Institute. Nature. https://doi.org/10.1038/ d41586-018-06656-y

Makarova KS, Aravind L, Grishin NV et al (2002) A DNA repair system specific for thermophilic Archaea and bacteria predicted by genomic context analysis. Nucleic Acids Res 30:482-496

Makarova KS, Grishin NV, Shabalina SA et al (2006) A putative RNA-interference-based immune system in prokaryotes: computational analysis of the predicted enzymatic machinery, functional analogies with eukaryotic RNAi, and hypothetical mechanisms of action. Biol Direct 1:7. https://doi. org/10.1186/1745-6150-1-7

Makarova KS, Aravind L, Wolf YI, Koonin EV (2011a) Unification of Cas protein families and a simple scenario for the origin and evolution of CRISPR-Cas systems. Biol Direct 6:38. https://doi. org/10.1186/1745-6150-6-38

Makarova KS, Haft DH, Barrangou R et al (2011b) Evolution and classification of the CRISPR-Cas systems. Nat Rev Microbiol 9:467-477. https://doi.org/10.1038/nrmicro2577

Makarova KS, Wolf YI, Alkhnbashi OS et al (2015) An updated evolutionary classification of CRISPRCas systems. Nat Rev Microbiol 13:722-736. https://doi.org/10.1038/nrmicro3569

McNutt M (2015) Breakthrough to genome editing. Science 350:1445. https://doi.org/10.1126/scien ce.aae 0479

Mohanraju P, Makarova KS, Zetsche B et al (2016) Diverse evolutionary roots and mechanistic variations of the CRISPR-Cas systems. Science 353:aad5147. https://doi.org/10.1126/science.aad5147

Mojica FJM, Díez-Villaseñor C, García-Martínez J, Soria E (2005) Intervening sequences of regularly spaced prokaryotic repeats derive from foreign genetic elements. J Mol Evol 60:174-182. https:// doi.org/10.1007/s00239-004-0046-3

Morange M (2015a) What history tells us XXXVII. CRISPR-Cas: the discovery of an immune system in prokaryotes. J Biosci 40:221-223

Morange M (2015b) What history tells us XXXIX. CRISPR-Cas: from a prokaryotic immune system to a universal genome editing tool. J Biosci 40:829-832

O'Malley MA, Koonin EV (2011) How stands the Tree of Life a century and a half after the origin? Biol Direct 6:32. https://doi.org/10.1186/1745-6150-6-32

Pourcel C, Salvignol G, Vergnaud G (2005) CRISPR elements in Yersinia pestis acquire new repeats by preferential uptake of bacteriophage DNA, and provide additional tools for evolutionary studies. Microbiol Read Engl 151:653-663. https://doi.org/10.1099/mic.0.27437-0

Pradeu T, Moreau JF (2018) CRISPR-Cas immunity: beyond nonself and defence. Biol Philos. https:// doi.org/10.1007/s10539-018-9665-8

Reardon S (2016) First CRISPR clinical trial gets green light from US panel. Nat News. https://doi. org/10.1038/nature.2016.20137

Shmakov S, Abudayyeh OO, Makarova KS et al (2015) Discovery and functional characterization of diverse class 2 CRISPR-Cas systems. Mol Cell 60:385-397. https://doi.org/10.1016/j.molce 1.2015 .10 .008

Veigl SJ (2018) A use/disuse paradigm for CRISPR-Cas systems. Biol Philos. https://doi.org/10.1007/ s10539-018-9661-Z

Wideman JG, Inkpen SA, Doolittle WF, Redfield RJ (2018) Mutationism, not Lamarckism, captures the novelty of CRISPR-Cas. Biol Philos. https://doi.org/10.1007/s10539-018-9659-6

Woolley S, Parke EC, Kelley D, Poole AM, Ganley A (2018) Striving for clarity about the "Lamarckian" nature of CRISPR-Cas systems. Biol Philos. https://doi.org/10.1007/s10539-018-9662-y 